\title{
Focus in Honor of David Muddiman, Recipient of the 2010 Biemann Medal
}

$\mathrm{T}^{\mathrm{s}}$

he 2010 Biemann Medal was awarded to Professor David C. Muddiman at the 58th annual ASMS Conference for his numerous research contributions to interdisciplinary bioanalytical mass spectrometry. This rather broad focus issue reflects Dave's own diverse research interests in the development of mass spectrometric instrumentation, methodologies, and their applications to biological problems as well as his long-term commitment to collaborative, crosscutting research. In particular, Dave has focused on innovative ways to create and manipulate ions to extend the usefulness of mass spectrometry by including new ionization methods for imaging mass spectrometry and improving ionization efficiency. These advances will ultimately allow for a greater understanding of the biology underlying complex molecular processes.

Dave was born in Long Beach, California, and grew up in a small community in Pennsylvania. He graduated from Gannon University in Erie, Pennsylvania in 1990, where his passion for scientific research was inspired by several dedicated professors who engaged Dave in undergraduate research. After graduation, Dave earned his Ph.D. in Chemistry in 1995 at the University of Pittsburgh under the direction of David M. Hercules, where he focused on the development of desorption ionization methods in conjunction with time-of-flight mass spectrometry for quantitative analysis. He joined the research group of Richard D. Smith at Pacific Northwest National Laboratory as a Department of Energy post-doctoral fellow, where he commenced investigations on nucleic acids by using electrospray ionization. In 1997, he joined the faculty at Virginia Commonwealth University as an assistant professor, where he forged a longlasting friendship with John Fenn, a landmark influence on Dave's development as a scientist, mentor, and leader. In 2002, he moved to the Mayo Clinic of Medicine in Rochester, Minnesota, where he established the Mayo Proteomics Research Center and several meaningful collaborations and friendships. Preferring the greater student interactions that an academic environment affords, Dave moved to North Carolina State University in 2005, where he is currently Professor of Chemistry and Director of the W. M. Keck FT-ICR Mass Spectrometry Laboratory. Dave was previously honored with an American Society for Mass Spectrometry Research Award in 1999 and the Arthur F.

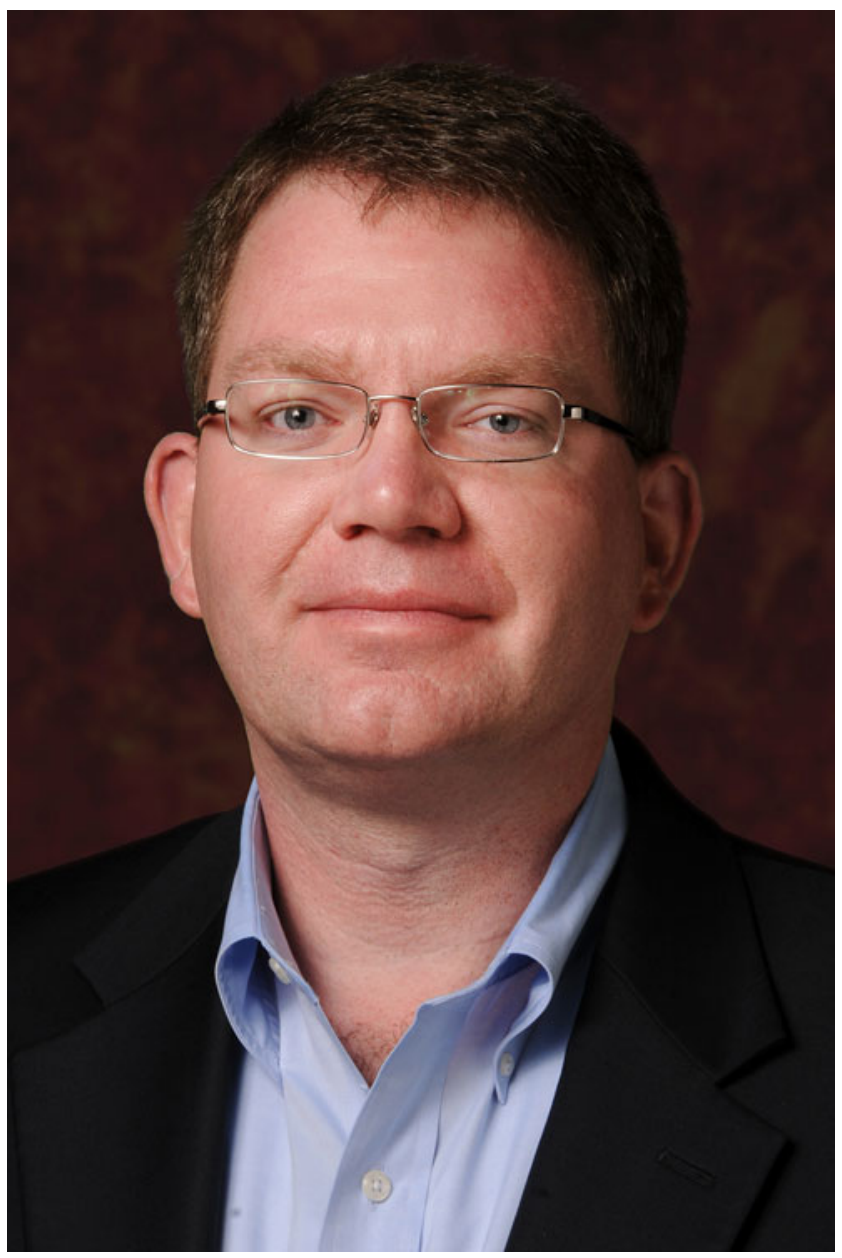

Findeis Award from the American Chemical Society in 2004.

This Special Focus Issue on "Bioanalytical Mass Spectrometry" is dedicated to Dave Muddiman. This issue opens with an Account and Perspective of John Fenn authored by Michael Grayson. John Fenn was one of Dave's special mentors due to their extensive interactions while on the faculty at Virginia Commonwealth University; John's broad outlook and passion for innovation inspired Dave's development as a leader in the field of mass spectrometry. This focus also contains original 
contributions from eight groups, including one by Dave's own research group. Dave and his co-authors report the synthesis and characterization of a series of hydrophobic reagents for derivatization of $N$-linked glycans and enhancement of their ion abundances upon electrospray ionization. In the third article, Richard D. Smith and coworkers describe the integration of subambient pressure nanoelectrospray ionization source with gradient reversedphase liquid chromatography, affording high sensitivity for complex mixtures like tryptic digests. Graham Cooks and co-authors report the development of desorption electrospray ionization (DESI) imaging for characterization of human seminoma tissue based on the glycerophospholipid profiles. Ljiljana Pasa-Tolic and co-workers calculate the electric field inside several FT-ICR cells and evaluate the outcome of cell compensation and impact on cell design. Kermit Murray and co-workers describe the use of an IR laser to ablate biological molecules from samples (blood, egg, milk) under ambient conditions, followed by capture of the ablated molecules in solvent droplets that are either deposited on a target and subsequently analyzed by MALDI or are injected into a nanoelectrospray source. Alan Marshall and co-workers present a re-designed FT-ICR mass spectrometer with improved conductance, better ion optical alignment, enhanced ion transmission, and reduced detection circuit capacitance, ultimately leading to a two-fold improvement in sensitivity. Pat Limbach and co-workers report an LCMS/MS method and selected reaction monitoring assay that enhances the identification and quantitative analysis of pseudouridine in RNA, as demonstrated for the incorporation of pseudouridine in the conserved T-loop of $E$. coli tRNA. In the final article of the special focus, Matthias Mann and co-workers report an innovative recalibration function that in some cases outperforms the use of a standard lock mass for improvement of mass accuracy in proteomics experiments utilizing an LTQOrbitrap mass spectrometer.

Dave is a rising star whose contributions to bioanalytical mass spectrometry have culminated in his selection as the recipient of the 2010 Biemann Medal. The Journal of the American Society for Mass Spectrometry is honored to present this focus section to highlight Dave's contributions and the significant impact of his interdisciplinary approach.

\author{
Jenny Brodbelt \\ Department of Chemistry and Biochemistry \\ University of Texas \\ Austin, TX, USA \\ e-mail: jbrodbelt@mail.utexas.edu
}

